
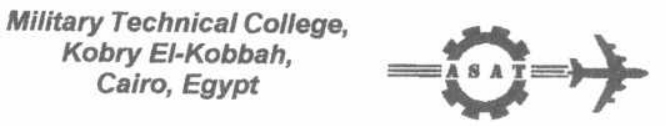

$9^{\text {th }}$ International Conference

On Aerospace Sciences \&

Aviation Technology

\title{
AN ADAPTIVE MULTI-TARGET TRACKING ESTIMATOR
}

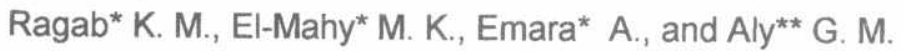

\begin{abstract}
Target tracking problem is normally composed of three main major functions: automatic track initiation, correlation, and track update. target tracking algorithms are usually based on applying optimal filtering theory. There have been numerous derivations of the optimal relations between the tracking parameters. In this paper, track update based on an adaptive Kalman algorithm with simplest case the adaptive $\alpha, \beta$ filter is formulated as shown in Fig.1. The necessary relations for pre-update extrapolation gain selection, update position, velocity filtering or smoothing, covariance matrix, and post-update are discussed. Implementation and results of multi-target tracking (MTT) using simulated electronic system input are presented. Results show a significant improvement in position and velocity of target tracking performance.
\end{abstract}

\section{KEY WORDS}

Multi-target Tracking, Signal Processing, and Kalman Filtering Theory.

\section{INTRODUCTION}

The number of the MTT applications $[1,2,3,4,5,6]$ is increasing rapidly with expansion in computer capabilities. Some of these applications include the development of sophisticated delivery system such as air traffic control (ATC). Many different approaches for MTT have been developed recently in response to the ever-increasing importance of the subject. So, non-standard approaches are accepted for all applications. Target tracking generates and maintains track data, which includes position and velocity. The major input to active tracking is electronic system data received from the radar input. This data includes positional information transformed to the systems coordinate axes and is referred to as plots. The active tracking is actually composed of three main major functions: automatic initiation, correlation and track update. Tracker is based on an adaptive Kalman algorithm with adaptive $\alpha, \beta$ filter [7] with addition of maneuver detection and smoothing.

* Egyptian Armed Forces,

** Faculty of Engineering, Ain-shams University. Cairo, Egypt. 


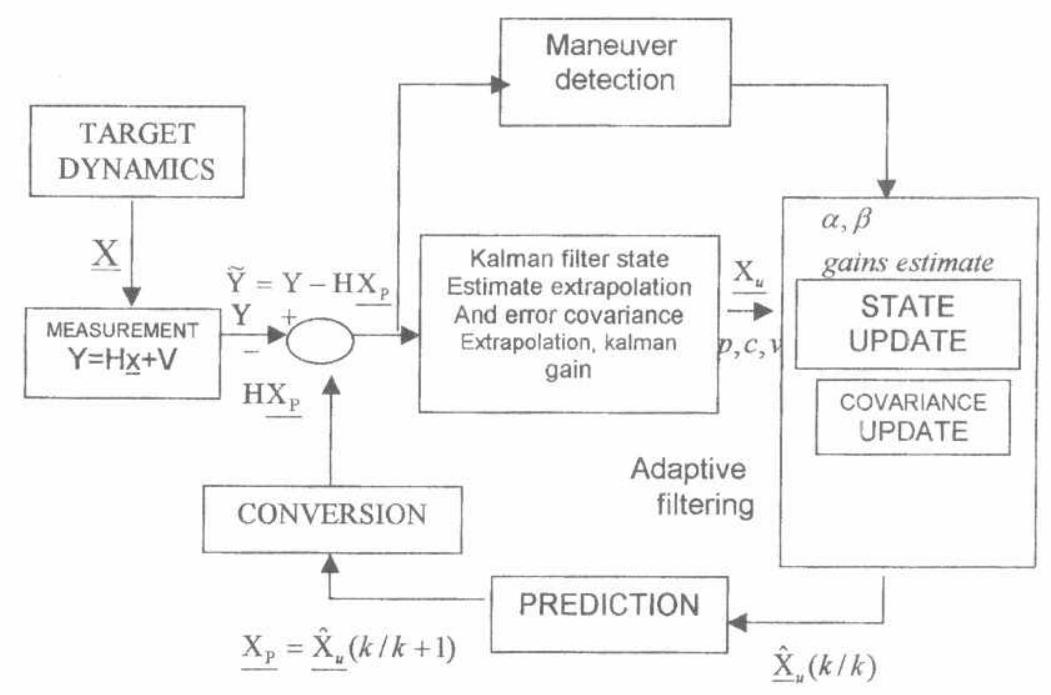

Fig.1. Filtering and prediction flow diagram.

\section{TRACKING ALGORITHM}

Tracking algorithm must be capable to following a maneuver target [9]. The Kalman filter models are optimally matched to target and measurement characteristics; therefore, any deviation from this state of affairs is an indication that these models are not correct [10]. If system model is not correct, track loss occurs easily. A range of adaptive algorithms has been developed to maintain tracking accuracy for a wide class of non-maneuver and maneuver target. Data filtering means smoothing a sequence of measurements and predicting future target kinematics behavior. The particular filter to be discussed here is the Kalman filter. It is the general solution to the recursive minimum mean-square error (MMSE) data-filtering problem within the class of linear estimators. In addition to minimizing the mean -square error the Kalman filter has a number of additional advantages if it is applied in the MTT. These advantages are included in the following properties $[3,7]$ : -

a. The gain sequence is chosen automatically, based on assumed target maneuver and measurement noise models. This means that same filter can be used for varying target and measurement environment by changing a few key parameters.

b. The Kalman gain sequence automatically adapts to the change in successive detection histories. This includes a varying sampling interval as well as missed detection.

c. The Kalman filter provides a convenient measure of the estimation accuracy though the covariance matrix. This measure required performing accurately the gating and the correlation functions.

d. Through the use of Kalman filter, it is possible to, at least partially, compensate for the effects of miscorrelation in the dense MTT environments. 


\subsection{Initiation}

Automatic initiation logic can be considered as Markov chain and the relevant performance data can be derived from its analysis in automatic initiation. There are two types of non-systems tracks: potential tracks and tentative tracks. For each received plot, we check the correlation with existing system tracks, tentative tracks, and existing potential tracks. If it is not correlated with all of them, then this plot will be a new potential track. When the correlation between this plot and potential tracks results in the formation tentative tracks [8], there are two consecutive hits and two out of four scan [10].

\subsection{Correlation}

Correlation is a process that attempts to associate each plot with existing track. An attempt is made to account for the presence of each received plot by correlating it with an existing system track. The correlation attempt starts by eliminating tracks that are very far from the plot to be considered. As results of the initial filtering called candidate track selection by testing correlation gate: initial check distance (ICD), non- maneuver and maneuver gates, and ambiguity resolution.

\section{TRACK UPDATE}

It is the third main major function of the tracking problem. It has the following steps:

- Pre-update extrapolation.

- Gain selection for Non-maneuver, Maneuver target.

- Update position, velocity filtering or smoothing and covariance matrix.

- Post-update extrapolation.

- Predict ion of the track position.

\section{PRE-UPDATE EXTRAPOLATION}

The kinematics models based on the Newton's law of motion can be used to describe the target motion where the target position evolves according to a polynomial in time in the absence of noise. Extrapolated target position depends on the track position, velocity, and correlation reference and plot time of detection $[4,7,8]-:$ -

$$
\begin{aligned}
& X_{U}=X_{E}+\left(V_{X} \Delta t\right) \\
& Y_{u}=Y_{E}+\left(V_{y} \Delta t\right)
\end{aligned}
$$

where $X_{u}, Y_{u}$ are the position of the track extrapolated., $X_{E}, Y_{E}$ are the track position at correlation reference time, $V_{X}, V_{Y}$ are the track velocity, $\Delta t=T-T_{\text {Ref, }} T$ is the plot detection time and $\mathrm{T}_{\text {Rer }}$ is the correlation reference time.

The plot measurement time is used to update the error covariance matrix $P$ :

$$
\begin{aligned}
& \mathrm{P}^{\prime}=\mathrm{P}+2 \cdot \delta \mathrm{T} \cdot \mathrm{C}+\mathrm{V} \cdot \delta \mathrm{T} \cdot \delta \mathrm{T} \\
& \mathrm{C}^{\prime}=\mathrm{C}+\delta \mathrm{T} \cdot \mathrm{V} \\
& \mathrm{V}^{\prime}=\mathrm{V}
\end{aligned}
$$

$P$ is the position error variance and $C$ is the position-velocity covariance, $V$ is velocity error variance, $\mathrm{P}^{\prime}$ is extrapolating position error variance, $\mathrm{C}^{\prime}$ is extrapolating velocity - 
position error covariance $V^{\prime}$ is extrapolating velocity error variance $\delta \mathrm{T}=\mathrm{T}-\mathrm{T}_{\mathrm{LS}}$, and $\mathrm{T}_{\mathrm{LS}}$ is the time of last smoothing for track.

\section{GAIN SELECTION}

The gains are computed based on the type of data whether are maneuver or non-nonmaneuver. The gain or the filter coefficients are determined for each track that participates in the active smoothing process. The gain consists of an $\alpha$ term used in smoothing track's position and $\beta / T$ term for smoothing the velocity.

\subsection{Non maneuver gain selection}

The non-maneuver-smoothing coefficient are calculated as $[4,8]$

$$
\begin{aligned}
\alpha & =\frac{P^{\prime}}{\left(P^{\prime}+M\right)} \\
\frac{\beta}{T} & =\frac{C^{\prime}}{\left(P^{\prime}+M\right)}
\end{aligned}
$$

$\alpha$ is Track positional smoothing parameter,

$\frac{\beta}{T}$ is Track velocity smoothing parameter

$M$ is the mean plot measuremen t error variance.

\subsection{Maneuver gain selection}

A- The filtering parameters $\alpha, \beta$ are selected from table.1 below using $\mathrm{R}$ Index [8]. The noise to maneuver ratio $R$ is defined as

$$
R=\frac{M^{1 / 2}}{T_{2} T_{\text {Max }}^{2}}
$$

where

$R$ is Noise - to - maneuver ratio, $\mathrm{T}_{\operatorname{Max}}=\operatorname{Max}\left(\delta \mathrm{T}, \mathrm{T}_{3}\right)$

$\delta \mathrm{T}=\mathrm{T}-\mathrm{T}_{\mathrm{LS}}$

$\mathrm{T}_{2}$ is Target maneuver level $(0.0026)$ this parameter have been identified by R. WENSKI [4].

$\mathrm{T}_{3}$ is Lower bound on time since smoothing

B- Convert $\beta$ from table to $\frac{\beta}{T}$ by dividing by $T_{\text {Max. }}$ 


\section{UPDATE TRACK POSITION AND VELOCITY SMOOTHING AND COVARIANCE MATRIX}

Smoothing or filtering data $\mathrm{X}_{u}, \mathrm{Y}_{u}$ is preformed depending on the obtained $\alpha, \beta$ as follows $[7,8]$.

Where

$$
\begin{aligned}
& X_{u}^{\prime}=X_{u}+\alpha \Delta X \\
& Y_{u}^{\prime}=Y_{u}+\alpha \Delta Y \\
& v_{X}^{\prime}=v_{X}+\left(\frac{\beta}{T}\right) \Delta X \\
& v_{y}^{\prime}=v_{y}+\left(\frac{\beta}{T}\right) \Delta Y
\end{aligned}
$$

$X_{u}^{\prime}, Y_{u}^{\prime}$ are smoothed position of the track at measurement time $\mathrm{v}_{\mathrm{x}}^{\prime}, \mathrm{v}_{\mathrm{y}}^{\prime}$ are Smoothed track velocity.

$X_{u}, Y_{u}$ are Position of the track extrapolated to measurement time. $\mathrm{v}_{\mathrm{x}}, \mathrm{v}_{\mathrm{y}}$ are Track velocity before smoothing.

$\Delta \mathrm{X}, \Delta \mathrm{Y}$ are plot_track $\mathrm{X}, \mathrm{Y}$ deviations.

Revise the track's error covariance matrix

$$
\begin{aligned}
& P=P^{\prime}-2 \alpha P^{\prime}+\alpha^{2}\left(P^{\prime}+M\right) \\
& C=(1-\alpha) C^{\prime}-\left(\frac{\beta}{T}\right) P^{\prime}+\alpha\left(\frac{\beta}{T}\right)\left(P^{\prime}+M\right) \\
& v=v^{\prime}-2\left(\frac{\beta}{T}\right) C^{\prime}+\left(\frac{\beta}{T}\right)^{2}\left(P^{\prime}+M\right)
\end{aligned}
$$

Where $P$ is the track revised position error variance, $C$ is the track revised position velocity variance, $v$ is the track revised velocity error variance $P^{\prime}, C^{\prime}, v^{\prime}$ are the track error terms extrapolated to time of update and $\left(\alpha, \frac{\beta}{T}\right)$ are smoothing coefficients.

\section{POST-UPDATE TRACK EXTRAPOLATION}

In maneuver tracking, Each track that has been smoothed with maneuver gate during the current correlation interval shall have its velocity component revised before post-update extrapolation, The position of the track shall be returned to the previous correlation reference time from the plot time of detection

$$
\begin{gathered}
\mathrm{X}_{\mathrm{E}}=X_{u}^{\prime}-\left(\mathrm{v}_{x} \Delta \mathrm{t}\right) . \\
\mathrm{Y}_{\mathrm{E}}=Y_{u}^{\prime}-\left(\mathrm{v}_{\mathrm{y}} \Delta \mathrm{t}\right) .
\end{gathered}
$$

where $X_{E}, Y_{E}$ are the smoothed track position extrapolated to the previous correlation reference time, $X_{u}, Y_{u}$ are the smoothed position of the track at measurement, $v_{x}, v_{y}$ are the newly smoothed track velocity $\left(v_{x}^{\prime}, v_{y}^{\prime}\right)$. 


\section{PREDICT TRACK POSITION}

The relation obtains the final prediction of the target position

$$
\begin{aligned}
& X_{p}=X+\left(v^{\prime} \Delta T_{p}\right) . \\
& Y_{p}=Y+\left(v_{y} \Delta T_{p}\right) .
\end{aligned}
$$

where $X_{p}, Y_{p}$ are the track position predicted current correlation reference time, $X, Y$ are the track position at the last estimates, $\Delta T_{p}$ is the difference between current and previous correlation reference time.

\section{SIMULATION AND RESULTS}

Programming language $\mathrm{C}++$ and Matlab Package are used for implementation and simulation. A "C++" program is designed for implementing a complete mult-target tracking (MTT) function. In this section, a complete structure of the MTT program is explained and it is shown how the main elements are connected together to give the final form of update track. First, electronic system signal processor sends data to the computer through a serial port. Data is received and stored into an input buffer file called "tp.in". The data are processed in first input and first output (FIFO) way.

The initial data are $P=0.35 n m i, C=0.29167 n m i, V=0.002430, V_{x}=0.055, V_{Y}=0.055$ scan time $=10 \mathrm{sec}, \mathrm{m}=0.35 \mathrm{nmi}$. Then, read the first plot from input file and make correlation between the initial track and first plot. If this plot is correlated with a track, perform track updating. The data generation steps are given by:

- Extrapolate the tracks position and updating error covariance matrix as shown in Table 4 and Equations 1, 2, 3, 4, and 5.

- Calculate the filter coefficient $\alpha, \beta$ for non-maneuver target as shown in Table 5 . for using Equations 6 and 7.

- Filtering coefficients $\alpha, \beta$ for maneuver target depending on calculating the maneuver index $(R)$ given in table 3,table 5 for using Equation 8.

- Select $\alpha, \beta$ from table 1 using $\mathrm{R}$ as shown in table.2and fig.3.

- Update track position and velocity, using Equations 9,10.11, and 12.

- Update track covariance matrix, as show in fig.5, table 6, using Equations 13,14, and 15.

- Calculate the predicted track positions, using Equations 18 and 19, the results are shown in Fig. 1,2,4.

\section{COMPARISONS}

The simulation shows that the performance of our proposed algorithm is better than the performance of Kalman filter algorithm $[3,4,7,8]$. The algorithm improved the target-target -tracking problem using an adaptive technique. The algorithm can be used for real time application without significant increasing in the complexity and formulas. 


\section{CONCLUSION}

The adaptive target track algorithm has been formulated using an adaptive Kalman algorithm with its simplest case an adaptive $\alpha, \beta$ filter. The algorithm achieve has efficient measurement error position and velocity. It also has reduced the processing time for real time application. The formulation of the tracking problem presented not only leads to results consistent parameters but also leads to know the tracking filter coefficient for maneuver or non-maneuvertarget. Therefore, the algorithm can be used to improve the track performance.

\section{REFERENCES}

[1] Wax, N.,"Signal-to-Noise Improvements and the Statistics of Tracking populations," Journal of Applied physics, Vol. pp. 586-595 (1955).

[2] Sittler, R.W., "An Optimal Data Association Problem in Surveillance theory," IEEE Transactions on Military Electronics, MIL-8, pp125-139, April (1964),

[3] H. C. Ramachadra "Kalman filtering techniques For radar tracking", Marcel Dekker, Inc., (2000).

[4] Williams, P.R "System Tracking Performance and Analysis" Ground systems group /Fullerton California, USA, Sep (1986).

[5] Jaffer, A.J., and Y. Bar Shalom, "On Optimal Tracking in Multiple Target Environments," Proceeding of the third symposium On Non-Linear Estimation theory and Its Applications, San Diego, CA, pp112-117 Sept. 11-13, (1972).

[6] Singer, R.A., R. Sea, and K.B Housewright, "Deviation and Evaluation of Improved Tracking Filters for Use in Dense Multi-Target Environments," IEEE Transactions on Information Theory, IT-20,, pp423-432, July (1974).

[7] BAYESIAN "Multiple Target Tracking" HC. Stone, L.D. AH Artech House Publishers. ,(1999).

[8] Dana, M.P., "Target Tracking Analysis" " Ground systems group /Fullerton California, USA, Aug(1986).

[9] Leard M; Leger J M ; and Brochard. "Trajectory Modeling and Target Tracking ", In The Twelfth IASTED International Conference, Modeling, Identification and Control, Page 119-122, Innsbruck, Austria, February (1993).

[10] Dana, M.P., "Establishment of Air Defense Sensor Requirements for Automatic Aircraft Tracking," AGARD Conference Proceedings No.252, Strategies for Automatic Track Initiation, Monterey, CA, pp. 19-1 to 19-20 Oct.(1978).

[11] Chang C B and Tabaczynsiki J A." Application of State Estimation to Target Tracking" IEEE Transactions on Automatic Control, AC-29, February, (1984).

[12] Bar-Shalom $Y$ and Fortmann TE. Tracking and Data Association, volume 179 of Mathematics in science and Engineering. Academic Press, Inc., USA, (1988). 
Table 1. Selection of $\alpha, \beta$ coefficients using $\mathrm{R}$ index.

\begin{tabular}{|c|c|c|}
\hline Noise-to-Maneuver- Ratio & $\alpha$ & $\beta$ \\
\hline $0<R \leq 0.55$ & 1 & 1 \\
$0.55<R \leq 1.27$ & 0.9 & 0.6 \\
$1.27<R \leq 2.39$ & 0.8 & 0.4 \\
$2.39<R \leq 3.94$ & 0.714 & 0.285 \\
$3.94<R \leq 5.98$ & 0.642 & 0.214 \\
$5.98<R$ & 0.583 & 0.166 \\
\hline
\end{tabular}

Table2. Evaluation $\alpha, \beta$ depends of noise to maneuver ratio $\mathrm{R}$

\begin{tabular}{|c|c|c|}
\hline$\beta$ & $\alpha$ & $\mathbf{R}$ \\
\hline 0.1666 & 1 & 0.39275 \\
\hline 0.1000 & 0.71428 & 1.02 \\
\hline
\end{tabular}

Table3. Evaluation noise to maneuver ratio $\mathrm{R}$ depends on mean plot error variance

\begin{tabular}{|l|l|}
\hline $\mathbf{R}$ & $\mathbf{M}$ \\
\hline 0.39275 & 0.35 \\
\hline 1.02 & 0.1509 \\
\hline 2.439033 & 0.85 \\
\hline
\end{tabular}

Table 4. Evaluation of the error position and velocity covariance matrices.

\begin{tabular}{|c|c|c|c|c|c||c|}
\hline \multicolumn{3}{|c|}{ Maneuve parameter(kalman) } & \multicolumn{3}{|c|}{ Non-maneuver parameter(kalman) } & \multirow{2}{*}{ Interval } \\
\hline $\boldsymbol{V}$ & $\boldsymbol{C}^{\prime}$ & $\boldsymbol{P}^{\mathbf{*}}$ & $\boldsymbol{V}^{\boldsymbol{N}}$ & $\boldsymbol{C}^{\prime}$ & $\boldsymbol{P}^{\prime}$ & \\
\hline 0.001431 & 0.05833 & 1.400009 & 0.002431 & 0.05833 & 1.40009 & 1 \\
\hline 0.002431 & 0.03281 & 0.7656 & 0.000486 & 0.01750 & 0.62999 & 2 \\
\hline 0.002431 & 0.031218 & 0.7273 & 0.000174 & 0.00833 & 0.39990 & 3 \\
\hline 0.002431 & 0.031118 & 0.7249 & 0.000081 & 0.00861 & 0.29166 & 4 \\
\hline 0.002431 & 0.031112 & 0.72480 & 0.000044 & 0.00318 & 0.22908 & 5 \\
\hline 0.002431 & 0.031111 & 0.72479 & 0.000027 & 0.00224 & 0.18845 & 6 \\
\hline
\end{tabular}


Table 5. Evaluation of the position and velocity filtering parameters.

\begin{tabular}{|c|c|c|c|c|c|}
\hline \multicolumn{3}{|c|}{ Maneuver gains } & \multicolumn{2}{|c|}{ Non-maneuver gains } & \\
\hline$R$ & $\beta$ & $\alpha$ & $\beta$ & $\alpha$ & \\
\hline \multirow[t]{6}{*}{0.3912} & 0.0500 & 0.9375 & 0.03333 & 0.80001 & Interval 1 \\
\hline & & & 0.01785 & 0.6428 & Interval 2 \\
\hline & & & 0.01111 & 0.5333 & Interval 3 \\
\hline & & & 0.00757 & 0.4545 & Interval 4 \\
\hline & & & 0.00545 & 0.3956 & Interval 5 \\
\hline & & & 0.004167 & 0.3499 & Interval 6 \\
\hline
\end{tabular}

Table6. Evaluation of the revised error position and velocity covariance matrix uses an adaptive algorithm.

\begin{tabular}{|c|c|c|c|c|c|c||}
\hline \multicolumn{2}{|c|}{ Maneuver Covariance matrix } & \multicolumn{2}{l||}{ Non-maneuver Covariance materix } & \multicolumn{1}{|c|}{} \\
\hline $\mathrm{V}$ & $\mathrm{C}$ & $\mathrm{P}$ & $\mathrm{V}$ & $\mathrm{C}$ & $\mathrm{p}$ & Interval \\
\hline 0.002431 & 0.00364 & 0.32812 & 0.000486 & 0.01166 & 0.28000 & 1 \\
\hline 0.002431 & 0.002051 & 0.32812 & 0.000174 & 0.006250 & 0.2250 & 2 \\
\hline 0.002431 & 0.001951 & 0.32812 & 0.000081 & 0.00388 & 0.1866 & 3 \\
\hline 0.002431 & 0.001945 & 0.32812 & 0.000044 & 0.00265 & 0.1590 & 4 \\
\hline 0.002431 & 0.001944 & 0.32812 & 0.000027 & 0.00192 & 0.1384 & 5 \\
\hline 0.002431 & 0.001944 & 0.32812 & 0.000017 & 0.00145 & 0.1224 & 6 \\
\hline
\end{tabular}




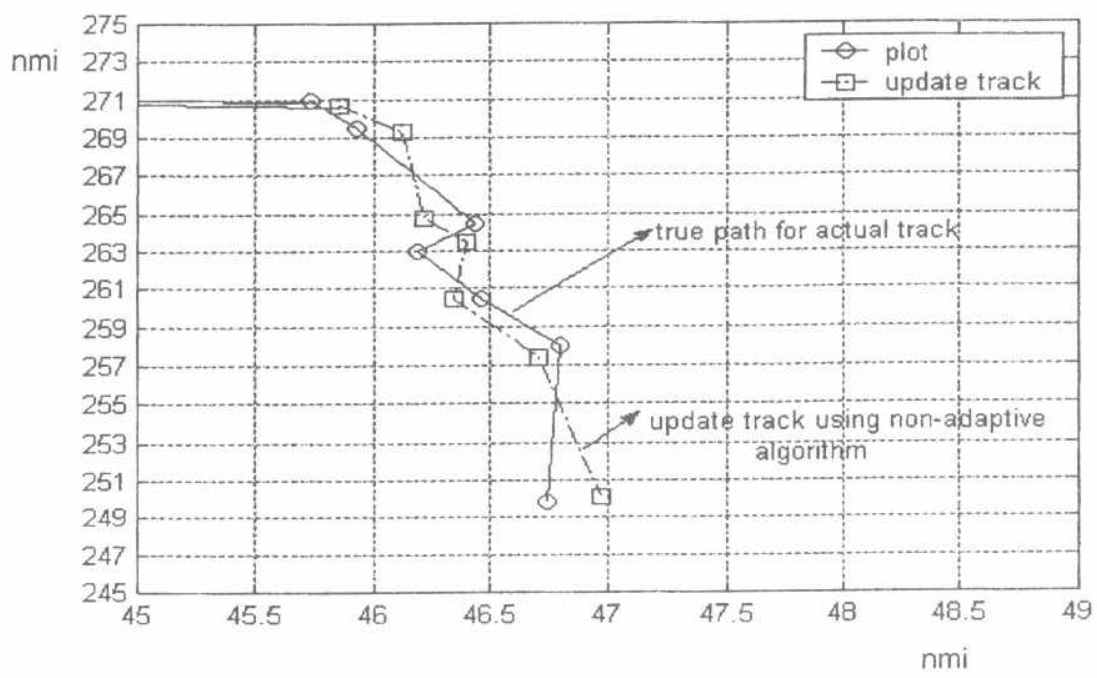

Fig.1.Maneuver $X-Y$ tracker performance using non-adaptive algorithm

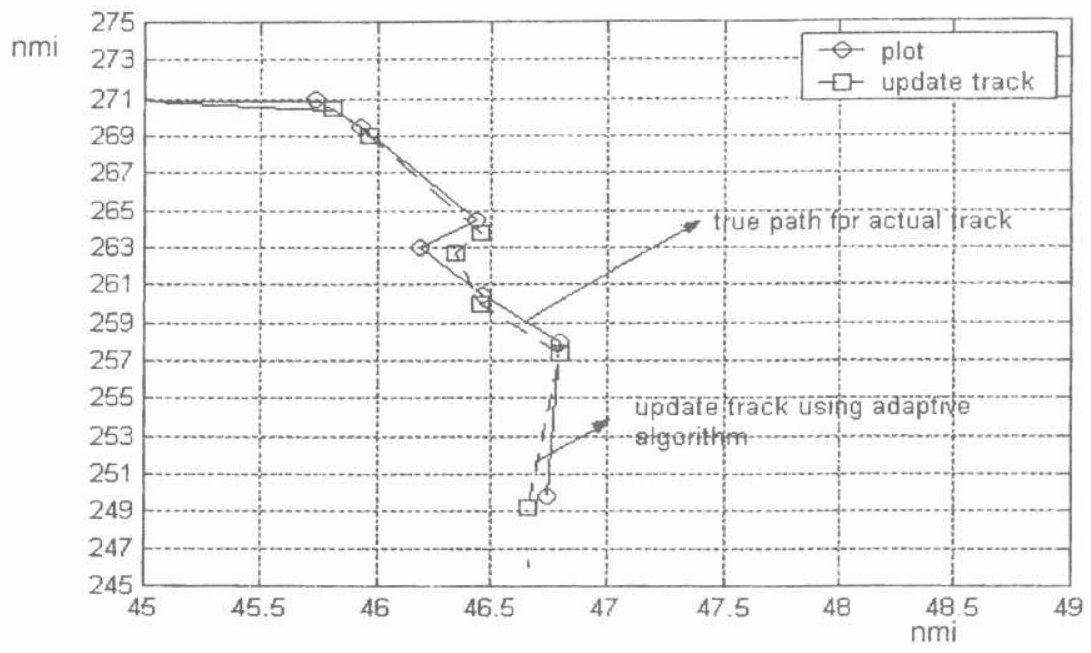

Fig. 2. Maneuver $X-Y$ tracker performance using an adaptive algorithm 

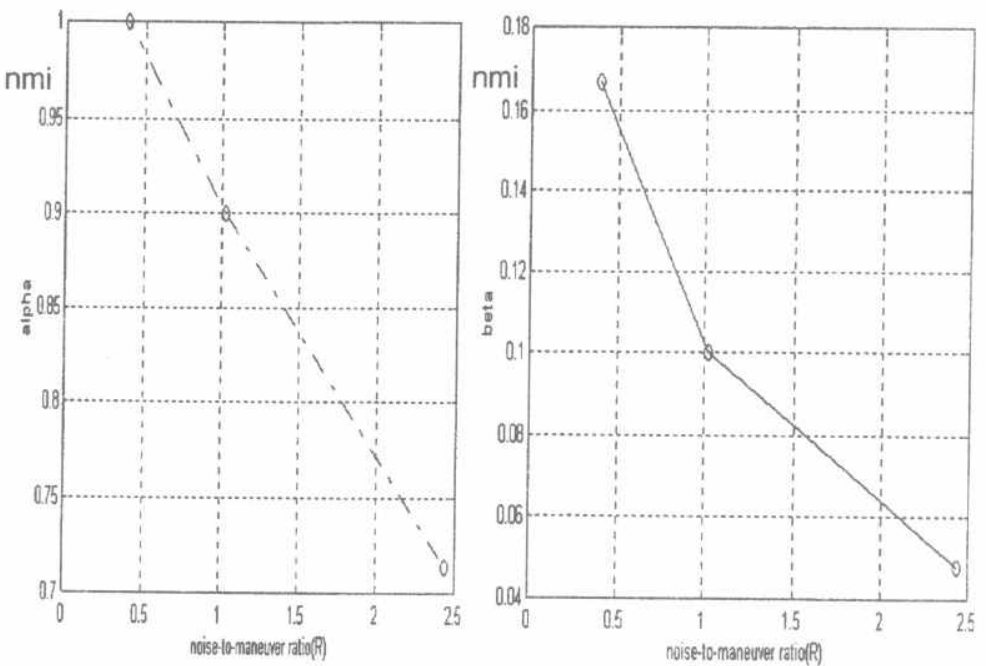

Fig.3. Smoothing parameter or gain coefficient depends on noise to maneuver ratio $R$

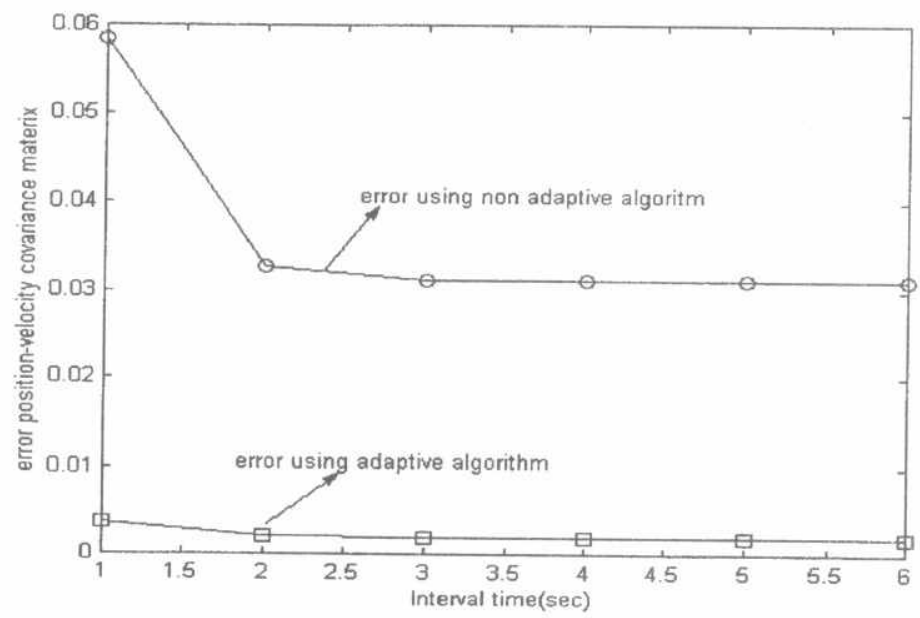

Fig.4. Error position- velcocity covariance matrerix using Kalman filter and adaptive algorithm 